\section{INTERACCIONES MEDICAMENTOSAS EN ANTIVIRALES PARA TRATAMIENTO DE LA COINFECCIÓN VIH/VHC EN PERÚ}

\author{
DRUG INTERACTIONS IN ANTIVIRALS FOR \\ THE TREATMENT OF HIVIVHC CO-INFECTION \\ IN PERU
}

\section{Jose A. Gonzales-Zamora ${ }^{1, a}$}

Sr. Editor. He leído con gran interés la norma técnica de reciente publicación sobre el manejo de la hepatitis $C$ en Perú, la cual está orientada a establecer los criterios técnicos para estandarizar el tamizaje, diagnóstico y tratamiento de la infección por el virus de la hepatitis $C$ $(\mathrm{VHC})^{(1)}$. Estos aspectos están muy bien desarrollados en la guía, especialmente para casos de monoinfección con VHC. No obstante, uno de los temas poco tratados es el manejo de la coinfección VIH/VHC. Cabe destacar que el manejo de esta población no difiere mucho de pacientes monoinfectados con VHC, sin embargo, en este contexto cobra gran importancia las interacciones medicamentosas entre los antivirales usados para tratar el VIH y el VHC.

La tabla redactada en la norma técnica detalla las interacciones medicamentosas en pacientes tratados con sofosbuvir/velpatasvir o glecaprevir/pibrentasvir, pero no se describe las interacciones con sofosbuvir/daclatasvir, siendo este un esquema de primera línea según la guía peruana ${ }^{(1)}$. Es necesario resaltar que sofosbuvir/daclatasvir no posee interacciones con la mayoría de inhibidores de la integrasa, sin embargo, los niveles de daclatasvir pueden incrementarse cuando se usa elvitegravir/ cobicistat, recomendándose en este caso disminuir la dosis de daclatasvir de $60 \mathrm{mg}$ a $30 \mathrm{mg}{ }^{(2)}$. Lo mismo sucede cuando se usa de conjuntamente con atazanavir/ ritonavir o atazanavir/cobicistat, ya que daclatasvir es metabolizado por el citocromo P450 (CYP) y es susceptible a interacciones con medicamentos inhibidores de esta enzima (2). Por otro lado, los niveles de daclatasvir pueden disminuir en presencia de medicamentos inductores del CYP3A4, como efavirenz y nevirapina, por los cual se recomienda incrementar la dosis de daclatasvir de $60 \mathrm{mg}$ a $90 \mathrm{mg}$ en presencia de estas drogas ${ }^{(2)}$ (Tabla 1). Una

\footnotetext{
1 Division of Infectious Diseases, Department of Medicine, University of Miami, Miller School of Medicine, Miami, Florida, USA

a Medical doctor

Recibido: 08/04/2019 Aprobado: 14/08/2019 En línea: 19/09/2019
}

Citar como: Gonzales-Zamora JA. Interacciones medicamentosas en antivirales para tratamiento de la coinfección VIH/VHC en Perú. Rev Peru Med Exp Salud Pública. 2019;36(3):537-9. doi: http://dx.doi.org/10.17843/ rpmesp.2019.363.4448 de las ventajas del esquema sofosbuvir/daclatasvir es la ausencia de interacción con tenofovir disoproxil fumarato (TDF), la cual constituye una droga de primera línea para el tratamiento de VIH en Perú. Asimismo, no se ha descrito interacciones importantes entre sofosbuvir/daclatasvir y darunavir/ritonavir o lopinavir/ritonavir.

Con referencia a sofosbuvir/velpatasvir, existen importantes interacciones medicamentosas sobre todo con efavirenz, el cual puede reducir significativamente los niveles de velpatasvir ${ }^{(3)}$. Efavirenz es un antiretroviral de primera línea en Perú, pero ya que su uso no está recomendado con sofosbuvir/velpatasvir, se sugiere en usuarios de esta droga, cambiar efavirenz a inhibidores de la integrasa o inhibidores de la proteasa. Lo mismo sucede con nevirapina, cuya utilización está contraindicada en presencia de velpatasvir. Según la norma técnica de tratamiento de VIH en Perú, lopinavir/ritonavir es una de las primeras alternativas de tratamiento en pacientes con contraindicación a efavirenz. Este inhibidor de la proteasa no tiene una interacción directa con sofosbuvir/velpatasvir, siendo una opción válida en este contexto. Sin embargo, velpatasvir podría incrementar los niveles de TDF sobretodo en esquemas en los que se utiliza ritonavir (3), por ello se recomienda una monitorización frecuente de la creatinina en estos pacientes, especialmente en aquellos con alto riesgo de falla renal (Tabla 1). Una estrategia comúnmente utilizada para evitar esta interacción es cambiar TDF a abacavir o tenofovir alafenamide, este último no disponible en el medio peruano.

Por lo descrito se infiere que los inhibidores de la integrasa son una excelente opción en pacientes tratados con sofosbuvir/velpatasvir, ya que no poseen interacciones medicamentosas. Lamentablemente estas drogas son todavía medicamentos de segunda línea en Perú, y requieren aprobación previa por el comité de expertos de $\mathrm{VIH}$, lo cual podría retrasar el tratamiento de pacientes infectados con VHC ${ }^{(4)}$.

Con respecto a glecaprevir/pibrentasvir, el uso concomitante de inhibidores de la proteasa está contraindicado, debido a que los niveles de glecaprevir/pibrentasvir pueden incrementarse significativamente produciendo hepatotoxicidad ${ }^{(5)}$. De igual manera, el uso de efavirenz o nevirapina no está recomendado, ya que reducen el nivel de glecaprevir/pibrentasvir, comprometiendo su efecto terapéutico ${ }^{(5)}$ (Tabla 1). En este contexto se prefieren los inhibidores de la integrasa en los esquemas antiretrovirales, ya que no existe una interacción significativa entre estos y glecaprevir/pibrentasvir. Afortunadamente, no se describen interacciones con los inhibidores nucleósidos de la transcriptasa reversa, incluido TDF.

Con relación a la monitorización del tratamiento, la norma técnica peruana recomienda el dosaje de carga viral para 
Tabla 1. Interacciones medicamentosas entre antivirales de acción directa y fármacos antirretrovirales

\begin{tabular}{|c|c|c|c|}
\hline $\begin{array}{l}\text { Antivirales de acción } \\
\text { directa para hepatitis C }\end{array}$ & $\begin{array}{c}\text { Clase de antirretroviral: } \\
\text { nombre del medicamento }\end{array}$ & $\begin{array}{l}\text { Efecto en la } \\
\text { concentración }\end{array}$ & Comentario \\
\hline \multirow{3}{*}{ Sofosbuvir/daclatasvir } & $\begin{array}{l}\text { Inhibidores de la proteasa: } \\
\text { Atazanavir/ritonavir } \\
\text { Atazanavir/cobicistat }\end{array}$ & $\uparrow$ daclatasvir & $\begin{array}{l}\text { La dosis de daclatasvir debe ser reducida a } 30 \mathrm{mg} \text { al } \\
\text { día cuando es administrada con atazanavir/ritonavir, } \\
\text { atazanavir/cobicistat u otro inhibidor fuerte del CYP3A4 }\end{array}$ \\
\hline & $\begin{array}{l}\text { INNTR: } \\
\text { Efavirenz } \\
\text { Nevirapina } \\
\text { Etravirina }\end{array}$ & $\downarrow$ daclatasvir & $\begin{array}{l}\text { La dosis de daclatasvir debe ser incrementada a } 90 \mathrm{mg} \\
\text { al dia cuando es administrada con efavirenz, etravirina, } \\
\text { nevirapina u otro inductor moderado del CYP3A4 }\end{array}$ \\
\hline & $\begin{array}{l}\text { Inhibidores de la integrasa: } \\
\text { Elvitegravir/cobicistat/FTC/ } \\
\text { TDF }\end{array}$ & $\uparrow$ daclatasvir & $\begin{array}{l}\text { La dosis de daclatasvir debe ser reducida a } 30 \mathrm{mg} \text { al día } \\
\text { cuando es administrada con cobicistat u otro inhibidor } \\
\text { fuerte del CYP3A4. }\end{array}$ \\
\hline \multirow{3}{*}{ Sofosbuvir/velpatasvir } & $\begin{array}{l}\text { Inhibidores de la proteasa: } \\
\text { Tipranavir/ritonavir }\end{array}$ & $\begin{array}{l}\downarrow \text { sofosbuvir } \\
\downarrow \text { velpatasvir }\end{array}$ & $\begin{array}{l}\text { La coadministración no ha sido bien estudiada, pero } \\
\text { puede disminuir los niveles de sofosbuvir y velpatasvir. } \\
\text { Su uso no está recomendado }\end{array}$ \\
\hline & $\begin{array}{l}\text { INNTR: } \\
\text { Efavirenz } \\
\text { Nevirapina }\end{array}$ & $\downarrow$ velpatasvir & $\begin{array}{l}\text { Los niveles de velpatasvir se reducen de forma } \\
\text { significativa cuando es administrado con efavirenz, } \\
\text { nevirapina u otro inductor moderado del CYP3A4. Su } \\
\text { uso concomitante con INNTR está contraindicado }\end{array}$ \\
\hline & $\begin{array}{l}\text { INTR: } \\
\text { Tenofovir disoproxil } \\
\text { fumarato }\end{array}$ & $\uparrow$ tenofovir & $\begin{array}{l}\text { Coadministración de velpatasvir con TDF incrementa } \\
\text { los niveles de TDF, sobre todo cuando se usa con } \\
\text { inhibidores de proteasa, por lo que se recomienda } \\
\text { monitorear la creatinina o cambiar TDF por abacavir o } \\
\text { tenofovir alafenamida fumarato (TAF) }\end{array}$ \\
\hline \multirow[t]{2}{*}{ Glecaprevir/pibrentasvir } & $\begin{array}{l}\text { Inhibidores de la proteasa: } \\
\text { Atazanavir } \\
\text { Darunavir } \\
\text { Lopinavir } \\
\text { Ritonavir }\end{array}$ & $\begin{array}{l}\uparrow \text { glecaprevir } \\
\uparrow \text { pibrentasvir }\end{array}$ & $\begin{array}{l}\text { Los niveles de glecaprevir y pibrentasvir se incrementan } \\
\text { de forma significativa cuando son administrados con } \\
\text { atazanavir, darunavir, lopinavir, ritonavir u otro inhibidor } \\
\text { del OATP1B, pudiendo producir hepatotoxicidad. Su } \\
\text { uso concomitante con inhibidores de la proteasa está } \\
\text { contraindicado }\end{array}$ \\
\hline & $\begin{array}{l}\text { INNTR: } \\
\text { Efavirenz } \\
\text { Nevirapina }\end{array}$ & $\begin{array}{l}\downarrow \text { glecaprevir } \\
\downarrow \text { pibrentasvir }\end{array}$ & $\begin{array}{l}\text { Los niveles de glecaprevir y pibrentasvir se reducen } \\
\text { significativamente cuando son administrados con } \\
\text { efavirenz, nevirapina u otro inductor del CYP3A4. Su uso } \\
\text { concomitante con INNTR está contraindicado }\end{array}$ \\
\hline
\end{tabular}

INNTR: inhibidores de la transcriptasa inversa no análogos de nucleósidos, INTR: inhibidores de la transcriptasa inversa análogos de los nucleósidos/ nucleótidos, FTC: Emtricitabina, TDF: Tenofovir disoproxil fumarato, CYP: citocromo P450, OATP: anión orgánico transportador de polipéptidos

VHC y transaminasas al inicio y 12 semanas después de culminado el tratamiento. Esta recomendación difiere de la guía norteamericana, la cual recomienda adicionalmente el monitoreo de la carga viral, transaminasas y creatinina luego de cuatro semanas de iniciado el tratamiento de hepatitis $C^{(6)}$. En cuanto a la función renal, la norma técnica peruana recomienda su monitoreo mensual en pacientes con filtración glomerular reducida que reciben sofosbuvir; sin embargo, en el contexto de coinfección VIH/VHC este monitoreo también está justificado en pacientes que reciben TDF e inhibidores de la proteasa conjuntamente con sofosbuvir/velpatasvir por el riego de nefrotoxicidad ${ }^{(6)}$.

La presencia de múltiples interacciones medicamentosas debe replantear el tratamiento de $\mathrm{VIH}$ en pacientes coinfectados con VHC en Perú, ya que el uso de efavirenz no constituye la mejor elección, prefiriéndose esquemas basados en inhibidores de la proteasa y sobre todo en inhibidores de la integrasa. Estos últimos son ideales por la ausencia de interacciones medicamentosas con los esquemas de tratamiento de hepatitis $C$ y por tener una tasa muy baja de efectos secundarios. Es probable que un mayor uso de inhibidores de integrasa en Perú pueda conllevar a un incremento del costo del tratamiento; sin embargo, el uso racional de estas drogas y su empleo temporal solo durante el tratamiento de VHC (de 8 a 12 semanas), permitiría un gasto poco significativo al sistema de salud público.

La aprobación de la norma técnica peruana constituye un hecho de suma importancia para el tratamiento de la 
hepatitis C. Su implementación permitirá el avance constante de esta área, sobre todo en el ámbito de la coinfección por $\mathrm{VIH}$ y VHC, la cual todavía se encuentra en desarrollo en Perú.

Fuentes de financiamiento: Ninguna

Conflictos de interés: El autor declara que no tiene conflictos de interés.

\section{REFERENCIAS BIBLIOGRÁFICAS}

1. MinisteriodeSalud. Resolución Ministerial N 1317-2018-MINSA. Norma Técnica de Salud para la Prevención, Diagnóstico y Tratamiento de la Hepatitis Viral C en el Perú. [Internet] Lima: MINSA; 2018 [citado 29 de marzo de 2019]. Disponible en: https://cdn.www.gob.pe/uploads/document/file/262935/ Resoluci\%C3\%B3n_Ministerial_N_1317-2018-MINSA.PDF

2. Daklinza (Daclatasvir)SummaryofProductCharacteristics[Internet]. Bristol-Myers Squibb; Montréal Bristol-Myers Squibb; 2014 [citado 20 de marzo de 2019]. Disponible en: https://www.bms.com/assets/ $\mathrm{bms} / \mathrm{ca} /$ documents/productmonograph/DAKLINZA_EN_ PM.pdf

3. EPCLUSA (Sofosbuvir and Velpatasvir) product monograph [Internet]. Gilead Sciences Ltd; 2016 [citado 10 de marzo de 2019). Disponible en: https://www.gilead.com/-/media/files/ pdfs/medicines/liver-disease/epclusa/epclusa_pi.pdf
4. MinisteriodeSalud. Resolución MinisterialN²15-2018-MINSA. Norma Técnica de Salud de Atención Integral del Adulto con Infección por el Virus de la Inmunodeficiencia Humana (VIH) [Internet]. Lima: MINSA; 2018 [citado 30 de marzo de 2019]. Disponible en: https://cdn.www.gob.pe/uploads/document/ file/187987/187482_R.M_215-2018-MINSA.PDF2018082324725-6ni25d.PDF

5. MAVYRET ${ }^{\mathrm{ix}}$ (Glecaprevir/Pibrentasvir) Prescribing Information, AbbVie Inc, 2017 [citado 29 de marzo de 2019. Disponible en: https://www.accessdata.fda.gov/drugsatfda_docs/ label/2017/209394s000lbl.pdf

6. Infectious Disease Society of America (IDSA); American Association for the Study of Liver Diseases (AASLD). HCV Guidance: Recommendations for Testing, Managing, and Treating Hepatitis C. [Internet]. Virginia: AASLD;2018 [citado 30 de marzo de 2019]. Disponible en: https://www. hcvguidelines.org/

Correspondencia: Jose A. Gonzales-Zamora

Dirección: 1120 NW 14th Street, Suite 863B. Miami, Fl 33136, USA

Teléfono: (001) 706-284-3510

Correoelectrónico:jxg1416@med.miami.edu 